EXTENDED REPORT

\title{
Ciprofloxacin susceptibility of Pseudomonas aeruginosa isolates from keratitis
}

\author{
J A Lomholt, M Kilian
}

Br J Ophthalmol 2003;87:1238-1240

See end of article for authors' affiliations .....................

Correspondence to: Jeanet A Lomholt, MD, $\mathrm{PhD}$ Department of Medical Microbiology and Immunology, University of Aarhus, Bartholin Building, Wilhelm Meyers allé, DK8000 Aarhus C, Denmark hans.lomholı@dadlnet.dk

Accepted for publication 6 February 2003

\begin{abstract}
Aim: To examine the ciprofloxacin susceptibility of 106 Pseudomonas aeruginosa eye isolates from the United Kingdom, Denmark, India, the United States, and Australia, and to determine the molecular mechanisms of resistance.

Methods: Ciprofloxacin susceptibility was tested by an agar dilution method; genomic DNA corresponding to the quinolone target genes gyrA and parC, and the regulatory genes mex $R$ and $n f x B$ controlling drug efflux systems, was amplified by PCR and sequenced; multilocus enzyme electrophoresis was performed to examine the genetic relation among resistant strains.

Results: Three out of 90 keratitis isolates (3.3\%), one from the United Kingdom and two from India, exhibited MIC values of $16 \mathrm{mg} / \mathrm{l}$ or $32 \mathrm{mg} / \mathrm{l}$. The UK isolate had a mutation in gyrA (Thr83lle), whereas the two Indian isolates showed mutations in both gyrA (Thr83lle) and parC (Ser87Leu). The remaining isolates from keratitis, endophthalmitis, contact lens associated red eye (CLARE), and contact lens storage cases showed MIC values below $1 \mathrm{mg} / \mathrm{l}$. Several allelic forms of gyrA and a single variation in the mexR gene product were detected in 10 ciprofloxacin susceptible strains.

Conclusions: The vast majority of eye isolates of $P$ aeruginosa from European countries are fully susceptible to ciprofloxacin and the concentration of ciprofloxacin eye drops used for local treatment (3000 mg/l) exceeds MIC values for strains recorded as resistant. Mutations in more than one target gene were associated with higher MIC values.
\end{abstract}

iprofloxacin monotherapy has proved effective in the treatment of keratitis ${ }^{1}$ and is now widely used as first

line treatment in many countries. In recent years, emerging ciprofloxacin resistance has been reported among Indian, Taiwanese, and American ocular Pseudomonas aeruginosa strains,,$^{2-5}$ causing concern to ophthalmologists worldwide. However, available data indicate that the prevalence of resistance among $P$ aeruginosa isolates varies between countries. Studies of $P$ aeruginosa isolates collected between 1994 and 1997 from Italian and Japanese hospitals showed that $83 \%$ and $90 \%$, respectively, were resistant. ${ }^{67}$ Recent population genetic analyses of clinical isolates of $P$ aeruginosa from various infections suggest that the majority of strains causing keratitis are distinct from strains from other infections. ${ }^{8}$

The main mechanism of quinolone resistance in $P$ aeruginosa is mutations in the genes gyrA and parC, encoding the target proteins DNA gyrase and topoisomerase IV, and mutations in the regulatory genes $m e x R$ and $n f x B$ for drug efflux pumps, resulting in reduced intracellular concentration. ${ }^{9}{ }^{10}$ However, the exact mechanisms of resistance vary among isolates from different infections. Jalal et a ${ }^{11}{ }^{12}$ found that mutations in $n f \times B$ are more common in resistant $P$ aeruginosa isolates from cystic fibrosis (CF) patients than in strains from urine and wounds in which mutations in gyrA and parC dominate. Japanese hospital isolates with high level of fluoroquinolone resistance showed a predominance of Thr83Ile mutation in gyrA. ${ }^{7}$ There is no information on resistance mechanisms in eye isolates.

The purpose of the study reported here was to determine the ciprofloxacin susceptibility of a collection of $P$ aeruginosa eye isolates from the United Kingdom, Denmark, United States, India, and Australia and to identify the mutations in target genes responsible for reduced susceptibility.

\section{METHODS}

The study included $106 P$ aeruginosa strains isolated between 1984 and 2001, the majority (96\%) after 1995. Ninety were isolated from keratitis, including 59 from the United Kingdom, 25 from Denmark, two from India, two from Australia, and two from the United States. The remaining strains were from endophthalmitis in the United Kingdom $(n=5)$, contact lens associated red eyes in India and Australia $(n=2)$, contact lens storage cases belonging to patients with keratitis in Denmark $(n=5)$, and contact lens cases belonging to asymptomatic wearers in India and Australia $(n=4)$. The quinolone susceptible reference strain PA01 was included for comparison.

\section{Minimal inhibitory concentration (MIC)}

The MIC for each strain was determined in triplicate using Isosensitest agar medium (Oxoid, Basingstoke, Hants, UK) containing ciprofloxacin in final concentrations of 32, 16, 8, $4,2,1,0.5,0.25,0.12,0.06$, and $0 \mu \mathrm{g} / \mathrm{ml} .^{12}$ The inoculum was about 100000 colony forming units transferred to the agar surface with a 96 pin applicator from a microtitration plate containing suspensions of the individual strains. The MIC was defined as the lowest concentration inhibiting visible bacterial growth as evaluated after 18 hour incubation at $37^{\circ} \mathrm{C}$. Using the reference value for resistance in systemic therapy, strains were regarded resistant to ciprofloxacin if MIC $\geqslant 2 \mathrm{mg} / \mathrm{l}$.

\section{Polymerase chain reaction (PCR)}

Chromosomal DNA was extracted as described. ${ }^{13}$ The quinolone resistance determining region of the genes encoding DNA gyrase (gyrA) and topoisomerase IV (parC), and the genes encoding regulatory genes for multidrug efflux pump systems MexAB-OprM and MexCD-OprJ, a 385 bp region of 
mexR and a 924 bp region including the whole $n f \times B$ gene $(561 \mathrm{bp})$, were amplified using PCR. The methods and primers used were essentially as described by Jalal et al ${ }^{14} 15$ except that only primers gyrA-1 and gyrA- 2 were employed to amplify the relevant stretch of the gyrA gene. All reactions were performed in Ready-To-Go PCR Beads (Amersham Pharmacia, Biotech Inc, Piscataway NJ, USA) added DNA and the two preboiled primers (10) each at a final concentration of $5 \mathrm{pmol} / \mu \mathrm{l}$. PCR products were sequenced on both strands using the same primers as employed in the PCRs with a Thermo Sequenase dye terminator cycle sequencing kit (Amersham Life Science, Cleveland, OH, USA) and analysis on an Applied Biosystem DNA sequencer. Numbering of altered amino acids is according to that in the complete proteins for strain PAOl (www.pseudomonas.com).

\section{Multilocus enzyme electrophoresis (MLEE)}

This method determines the electrophoretic mobilities of housekeeping enzymes, ${ }^{14}$ and was performed to examine the genetic relation among the resistant strains. The electrophoretic mobility of nine enzymes (malate dehydrogenase, alkaline phosphatase, glutamate dehydrogenase, glucose-6phosphate dehydrogenase, esterases, phosphoglucose isomerase, leucine isomerase, hexokinase, and alcohol dehydrogenase) was determined as described. ${ }^{8}$

\section{RESULTS}

The triplicate analysis of the ciprofloxacin susceptibility of each strain revealed excellent reproducibility. A total of 104 isolates showed identical results in all three determinations or variations within a single step dilution. Two isolates showed variations within two dilutions.

Of the $106 P$ aeruginosa isolates, three $(2.8 \%)$ showed in vitro resistance to ciprofloxacin. The resistant isolates were all isolated from keratitis, two out of two isolates from India, and one out of 59 isolates from the United Kingdom The two strains from India exhibited median MICs of $32 \mathrm{mg} / \mathrm{l}$ and the strain from the United Kingdom showed a median MIC of 16 $\mathrm{mg} / \mathrm{l}$.

The median MIC of ciprofloxacin for the remaining strains varied between $0.12-0.25 \mathrm{mg} / \mathrm{l}$ for 100 isolates, was $0.5 \mathrm{mg} / \mathrm{l}$ for three isolates, and $1 \mathrm{mg} / \mathrm{l}$ for one strain (PA01); $95 \%$ of the strains showed median MIC values identical to or below $0.25 \mathrm{mg} / \mathrm{l}$.

Mutations in target genes were found in all three resistant isolates (Table 1). All three had a mutation in gyrA, corresponding to amino acid change Thr83Ile. The two Indian isolates (paer31 and paer32), in addition, showed a mutation in parC corresponding to amino acid change Ser87Leu. The additional mutation in these two strains was associated with a higher MIC value $(32 \mathrm{mg} / \mathrm{l}$ compared with $16 \mathrm{mg} / \mathrm{l}$ for strain MK56). None of the strains had mutations in $n f x B$.

Sequence analysis of the corresponding segments of gyrA, parC, and mexR in 10 fully susceptible isolates that were phylogenetically related to the resistant strains ${ }^{8}$ revealed several allelic forms of gyrA and a single variation in the mexR gene product. The following non-synonymous mutations relative to PAOl were found in GyrA: Alal 17Pro (GCC $\rightarrow$ CCC in strains AKH01, MK58, PJ39), Arg121Gln (CGA $\rightarrow$ CAA in MK30, MK34, MK56 (resistant), MK58, Leul25Val (TTG $\rightarrow$ GTG in all strains except for PAOl and the resistant strain paer32). The variation observed in MexR was: Phe80Val (TTC $\rightarrow$ GTC in all strains except for PAOl, MK2, and PJ39). None of the two mutations present in resistant strains was detected in the 10 susceptible strains.

The electrophoretic mobilities of the nine housekeeping enzymes from paer31 and paer32 were identical, strongly suggesting that they belong to the same clone. The UK isolate MK56 showed different mobilities for the esterases, glucose6-phosphate dehydrogenase, and leucine aminopeptidase, indicating that this strain is clonally unrelated to the two resistant isolates from India.

\section{DISCUSSION}

This study shows that ciprofloxacin resistance is still rare among $P$ aeruginosa isolates from eye infections in Europe. Only one out of 102 isolates $(0.98 \%)$ from Europe revealed an MIC value of $16 \mathrm{mg} / \mathrm{l}$, thus exceeding the value normally regarded as reference value for resistance in systemic therapy (MIC $\geqslant 2 \mathrm{mg} / \mathrm{l}$ ). The finding that both of two isolates from India were resistant is in accordance with previous studies that revealed a resistance rate of $15.6-30.7 \%$ among eye isolates in India. ${ }^{215}$ Significantly lower rates have been observed in the United States $(2.1 \%)$ and Japan $(3.4 \%){ }^{4}{ }^{7}$ Conceivably, differences in susceptibility between countries and between different environments within countries reflect different degrees of selection pressures. In contrast with nosocomially acquired $P$ aeruginosa infections, eye infections arise in healthy contact lens wearers in the community with less selection for resistance and with clonally distinct isolates. ${ }^{8}$

Evaluation of the susceptibility of $P$ aeruginosa keratitis isolates is usually made with reference to concentrations used in systemic therapy. Nevertheless, the concentrations achieved on the ocular surface by local application of antibiotic eye drops or ointments are significantly higher. ${ }^{16}{ }^{17}$ The concentration of ciprofloxacin in eye drops used for local treatment $(3000 \mathrm{mg} / \mathrm{l})$ far exceeds in vitro MIC values (16$32 \mathrm{mg} / \mathrm{l}$ ) even for the three strains recorded as resistant in this study. Whether the increased resistance of bacteria in biofilms will affect their susceptibility to treatment in vivo is not clear.

The three eye isolates with reduced susceptibility had previously recognised mutations in different combinations of target genes. Mutations in more than one gene were associated with a higher level of resistance. The frequently observed mutation at position 83 in gyrA, associated with a high level of resistance in $P$ aeruginosa, Escherichia coli, and Yersinia pestis, was found in all three isolates. ${ }^{70}{ }^{18}$ In addition,

Table 1 Mutations detected in fluoroquinolone resistant strains of Pseudomonas aeruginosa in the target genes gyrA and parC

\begin{tabular}{llll}
\hline & & \multicolumn{2}{c}{ Amino acid change (nucleotide change) } \\
\cline { 4 - 5 } Isolate & MIC $(\mathrm{mg} / \mathrm{ll})$ of ciprofloxacin & gyrA & parC \\
\hline PAO1 & 1.0 & T83I (ACC $\rightarrow$ ATC) & \\
MK 56 & 16 & T83I (ACC $\rightarrow$ ATC) & S87L (TCG $\rightarrow$ TTG) \\
Paer 31 & 32 & T83I (ACC $\rightarrow$ ATC) & S87L (TCG $\rightarrow$ TTG) \\
Paer 32 & 32 & &
\end{tabular}

$\mathrm{L}=$ leucine; $\mathrm{S}=$ serine $; \mathrm{I}=$ isoleucine $; \mathrm{T}=$ threonine; $\mathrm{K}=$ lysine; $\mathrm{F}=$ phenylalanine; $\mathrm{E}=$ glutamic acid; $\mathrm{N}=$ asparagine; $\mathrm{A}=$ alanine. All changes are relative to reference strain PAO1, which is quinolone susceptible. 
the two Indian strains shared a previously described mutation in parC (Ser87Leu). GyrA and ParC with these two mutations show an increased ability to supercoil DNA in the presence of ciprofloxacin. ${ }^{19}$ The finding of identical mutations in the two Indian isolates is in agreement with their clonal identity as revealed by MLEE analysis. Sequence analysis of 10 additional isolates revealed several allelic variations in gyrA that were not associated with decreased susceptibility to ciprofloxacin.

In conclusion, ciprofloxacin resistance of $P$ aeruginosa eye isolates from countries with a restrictive usage of antibiotics is still rare and the problem is considerably smaller than that observed in certain hospital environments. These findings are relevant also for the third and fourth generation fluoroquinolones, as there is cross resistance between ciprofloxacin and these new compounds among Gram negative bacteria. ${ }^{20}$

\section{ACKNOWLEDGEMENT}

This study was supported by Alcon Denmark.

\section{Authors' affiliations}

J A Lomholt, M Kilian, Department of Medical Microbiology and Immunology, University of Aarhus, DK-8000 Aarhus C, Denmark

\section{REFERENCES}

1 Cokingtin CD, Hyndiuk RA. Insights from experimental data on ciprofloxacin in the treatment of bacterial keratitis and ocular infections. Am J Ophthalmol 1991; 112(Suppl 4):25S-28S.

2 Garg P, Sharma S, Rao GN. Ciprofloxacin-resistant Pseudomonas keratitis. Ophthalmology 1999;106:1319-23.

3 Hu FR, Luh KT. Pseudomonas aeruginosa isolated from corneal ulcer: susceptibility to antimicrobial agents tested alone or in combination. J Formos Med Assoc 1992;91:190-4.

4 Chaudhry NA, Flynn HW Jr, Murray TG, et al. Emerging ciprofloxacinresistant Pseudomonas aeruginosa. Am J Ophthalmol 1999;128:105-10.

5 Vajpayee RB, Dada T, Saxena R, et al. Study of the first contact management profile of cases of infectious keratitis: a hospital-based study. Cornea 2000;19:52-6.
6 Segatore B, Setacci D, Perilli $M$, et al. Italian survey on comparative levofloxacin susceptibility in 334 clinical isolates of Pseudomonas aeruginosa. Antimicrob Agents Chemother 1999;43:428-31.

7 Takenouchi T, Sakagawa E, Sugawara M. Detection of gyrA mutations among 335 Pseudomonas aeruginosa strains isolated in Japan and their susceptibilities to fluoroquinolones. Antimicrob Agents Chemother 1999;43:406-9.

8 Lomholt JA, Poulsen K, Kilian M. Epidemic population structure of Pseudomonas aeruginosa: evidence for a clone that is pathogenic to the eye and that has a distinct combination of virulence factors. Infect Immun 2001;69:6284-95.

9 Drlica K, Zhao X. DNA gyrase, topoisomerase IV, and the 4-quinolones. Microbiol Mol Biol Rev 1997;61:377-92.

10 Kureishi A, Diver JM, Beckthold B, et al. Cloning and nucleotide sequence of Pseudomonas aeruginosa DNA gyrase gyrA gene from strain PAOI and quinolone-resistant clinical isolates. Antimicrob Agents Chemother 1994;38:1944-52.

11 Jalal S, Wretlind B. Mechanisms of quinolone resistance in clinical strains of Pseudomonas aeruginosa. Microb Drug Resist 1998;4:257-61.

12 Jalal S, Ciofu O, Høiby N, et al. Molecular mechanisms of fluoroquinolone resistance in Pseudomonas aeruginosa isolates from cystic fibrosis patients. Antimicrob Agents Chemother 2000;44:710-12.

13 Poulsen K, Hjorth JP, Kilian M. Limited diversity of the immunoglobulin A1 protease gene (iga) among Haemophilus influenzae serotype b strains. Infect Immun 1988;56:987-92.

14 Selander RK, Caugant DA, Ochman H, et al. Methods of multilocus enzyme electrophoresis for bacterial population genetics and systematics. Appl Environ Microbiol 1986:51:873-84.

15 Kunimoto DY, Sharma S, Garg P, et al. In vitro susceptibility of bacterial keratitis pathogens to ciprofloxacin. Emerging resistance. Ophthalmology 1999;106:80-5.

16 Shell JW. New systems for the ocular delivery of drugs. In: Johnson P, LloydJones LG, eds. Drug delivery systems. Chichester: Ellis Horwood, 1987:243-66.

17 Lomholt JA, Møller JK, Ehlers N. Prolonged persistence on the ocular surface of fortified gentamicin ointment as compared to fortified gentamicin eye drops. Acta Ophthalmol Scand 2000;78:34-6.

18 Lindler LE, Fan W, Jahan N. Detection of ciprofloxacin-resistant Yersinia pestis by fluorogenic PCR using the LightCycler. J Clin Microbiol 2001;39:3649-55.

19 Akasaka T, Tanaka M, Yamagushi A, et al. Type II topoisomerase mutations in fluoroquinolone-resistant clinical strains of Pseudomonas aeruginosa isolated in 1998 and 1999: role of target enzyme in mechanisms of fluoroquinolone resistance. Antimicrob Agents Chemother 2001;45:2263-8.

20 Mather R, Karenchak LM, Romanowski EG, et al. Fourth generation fluoroquinolones: new weapons in the arsenal of ophthalmic antibiotics. Am J Ophthalmol 2002;133:463-6. 